\title{
Stabilizing quantum metastable states in a time-periodic potential
}

\author{
Choon-Lin Ho \\ Department of Physics, Tamkang University, Tamsui 25137, Taiwan \\ Chung-Chieh Lee \\ Department of Electronic Engineering, Tung Nan Institute of Technology, Shenkeng, Taipei 222, Taiwan
}

\begin{abstract}
In this talk we present a model to demonstrate how time-periodic potential can be used to manipulate quantum metastability of a system. We study metastability of a particle trapped in a well with a time-periodically oscillating barrier in the Floquet formalism. It is shown that the oscillating barrier causes the system to decay faster in general. However, avoided crossings of metastable states can occur with the less stable states crossing over to the more stable ones. If in the static well there exists a bound state, then it is possible to stabilize a metastable state by adiabatically increasing the oscillating frequency of the barrier so that the unstable state eventually cross-over to the stable bound state. It is also found that increasing the amplitude of the oscillating field may change a direct crossing of states into an avoided one. Hence, one can manipulate the stability of different states in a quantum potential by a combination of adiabatic changes of the frequency and the amplitude of the oscillating barrier.
\end{abstract}

\section{INTRODUCTION}

Ever since the advent of quantum mechanics, quantum tunneling has been an important and fascinating subject. This phenomenon arises frequently in physics. In fact, one of the first successful applications of quantum mechanics has been the explanation of the $\alpha$-decay of atoms as a quantum tunneling process [1]. Recent examples include tunneling phenomena in semiconductors and superconductors [2], in Josephson junction systems [3], resonant tunneling in heterojunction nanostructures [4], tunneling ionization of atoms [5], photon-assisted tunneling in superconducting junctions and semiconductor superlattices [6], etc.

In cosmology, quantum metastable states play an essential rôle in some versions of the inflationary models of the early universe 7]. In these models inflation of the early universe is governed by a Higgs field trapped in a metastable state. Inflation ends when the metastable state decays to the true ground state of the universe. During inflation the universe expands exponentially. It is thus obvious that the metastable state of the Higgs field is trapped in a rapidly varying potential. The problem is therefore a truly time-dependent one. Unfortunately, owing to the inherent difficulties of the problem, more often than not one has to consider the decay of the Higgs field in a quasi-stationary approximation, in which the decay is studied assuming a static potential [8]. Before one can deal with the decay of Higgs field in the non-stationary potential, it is desirable to gain some insights first by studying metastability in time-dependent potential in simple quantum-mechanical models.

An early attempt at studying the effects of time-varying forces on quantum metastability appears in Fisher's work [9], which was motivated by an experiment on quantum tunneling of the phase in a current-biased Josephson junction with a weak microwave perturbation [10]. In this work Fisher considered the general problem of quantum tunneling in a metastable well with a weak oscillatory force. There he reformulated the standard WKBJ approach to quantum decay in order to include a weak time-dependent perturbation. For a class of metastable potentials which interpolates between the cubic potential and a truncated harmonic-oscillator potential, he showed that the decay rate is generally enhanced by the weak oscillatory force. The potential considered by Fisher has a number of oscillator-like levels near its minimum. The opposite situation where only two levels are present was considered by Sokolovski [11], who studied the effect of a small AC field mixing two levels in the well on the tunneling rate in a semiclassical framework.

The results in [9, 11] are quite general for a class of weak oscillatory forces. However, it is desirable to consider other possibilities, e.g., exact solutions and/or nonperturbative results.

In [12, 13] we have considered simple driven quantum metastable models in which a particle is trapped in a well with a periodically driven rectangular barrier. In order to do away with any restriction of amplitude or frequency of the driving force, and of the number of states in the potential, we treat the problems in the framework of the Floquet formalism [14, 15, 16]. An exact expression determining the Floquet quasi-energies of stable/metastable states in the well is derived. From the solution of this equation we find that while the oscillating barrier makes the system decay faster in general, there is the possibility that avoided crossings of metastable states can occur with the less stable states crossing over to the more stable ones.

That an oscillating potential can affect the tunneling property of a system has also been noticed before, eg. in quantum transport process [16, 17] ). In [16] it was demonstrated that an propagating particle at appropriate incident 
energy can be trapped into a bound state by an oscillating square-well. In [17] it was found that a particle can be localized in one side of a time-dependent double well if the amplitude and the frequency of the oscillating field were chosen properly. Our examples shows how a time-periodic field can modify the metastability of a decaying state.

\section{THE MODEL}

To be concrete, in this talk we present the model considered in [12]. This model consists of a particle of mass $m$ trapped in a square well with a harmonically oscillating barrier,

$$
V(x, t)= \begin{cases}\infty, & x<0 \\ 0, & 0 \leq x<a \\ V_{0}+V_{1} \cos (\omega t), & a \leq x \leq b \\ V_{0}^{\prime}, & x>b\end{cases}
$$

Here $V_{0}, V_{1}, V_{0}^{\prime}$ and $\omega$ are positive parameters, with $V_{0}^{\prime}<V_{0}$ and $V_{1}<V_{0}-V_{0}^{\prime}$. According to the Floquet theorem, the wave function of a time-periodic system has the form $\Psi_{\varepsilon}(x, t)=e^{-i \varepsilon t / \hbar} \Phi_{\varepsilon}(x, t)$, where $\Phi_{\varepsilon}(x, t)$ is a periodic function with the period $T=2 \pi / \omega$, i.e. $\Phi_{\varepsilon}(x, t+T)=\Phi_{\varepsilon}(x, t)$, and $\varepsilon$ is the Floquet quasi-energy, which we will call Floquet energy for brevity. It should be noted that the Floquet energy is determined only modulo $\hbar \omega$. For if $\left\{\varepsilon, \Phi_{\varepsilon}\right\}$ is a solution of the Schrödinger equation, then $\left\{\varepsilon^{\prime}=\varepsilon+n \hbar \omega, \Phi_{\varepsilon^{\prime}}=\Phi_{\varepsilon} \exp (i n \omega t)\right\}$ is also a solution for any integer $n$. But they are physically equivalent as the total wave function $\Psi_{\varepsilon}$ is the same [15]. All physically inequivalent states can be characterized by their reduced Floquet energies in a zone with a width $\hbar \omega$. We therefore consider solutions of $\varepsilon$ only in the first Floquet zone, i.e. $\varepsilon \in[0, \hbar \omega)$.

The wave function of the particle is described as follows [12]:

$$
\begin{aligned}
\Psi(x, t) & =e^{-i \varepsilon t / \hbar} \Phi_{\varepsilon}(x, t) \\
& =e^{-i \varepsilon t / \hbar} \begin{cases}\sum_{n=-\infty}^{\infty} A_{n} \sin \left(k_{n} x\right) e^{-i n \omega t}, & 0 \leq x<a, \\
\sum_{n=-\infty}^{\infty} \sum_{l=-\infty}^{\infty}\left(a_{l} e^{q_{l} x}+b_{l} e^{-q_{l} x}\right) J_{n-l}\left(\frac{V_{1}}{\hbar \omega}\right) e^{-i n \omega t}, & a \leq x \leq b, \\
\sum_{n=-\infty}^{\infty} t_{n} e^{i k_{n}^{\prime} x} e^{-i n \omega t}, & x>b,\end{cases}
\end{aligned}
$$

where

$$
\begin{aligned}
k_{n} & =\sqrt{2 m(\varepsilon+n \hbar \omega)} / \hbar, \\
q_{l} & =\sqrt{2 m\left(V_{0}-\varepsilon-l \hbar \omega\right)} / \hbar, \\
k_{n}^{\prime} & =\sqrt{2 m\left(\varepsilon+n \hbar \omega-V_{0}^{\prime}\right)} / \hbar,
\end{aligned}
$$

and $J_{n}$ 's are the Bessel functions. In the region $x>b$, we have adopted Gamow's outgoing boundary condition, namely, there is no particle approaching the barrier from the right [1]. Matching the wave function and its first derivative at the boundaries $x=a$ and $x=b$, we obtain the relations among the coefficients $A_{n}, a_{n}, b_{n}$ and $t_{n}$ :

$$
\begin{aligned}
A_{n} \sin \left(k_{n} a\right) & =\sum_{l}\left(a_{l} e^{q_{l} a}+b_{l} e^{-q_{l} a}\right) J_{n-l}(\alpha), \\
k_{n} A_{n} \cos \left(k_{n} a\right) & =\sum_{l} q_{l}\left(a_{l} e^{q_{l} a}-b_{l} e^{-q_{l} a}\right) J_{n-l}(\alpha), \\
t_{n} e^{i k_{n}^{\prime} b} & =\sum_{l}\left(a_{l} e^{q_{l} b}+b_{l} e^{-q_{l} b}\right) J_{n-l}(\alpha), \\
i k_{n}^{\prime} t_{n} e^{i k_{n}^{\prime} b} & =\sum_{l} q_{l}\left(a_{l} e^{q_{l} b}-b_{l} e^{-q_{l} b}\right) J_{n-l}(\alpha),
\end{aligned}
$$

where $\alpha \equiv V_{1} / \hbar \omega$. The Floquet energy is determined from these relations by demanding non-trivial solutions of the coefficients. In practice, however, we must truncate the above equations to a finite number of terms, or sidebands as are usually called in the literature, eg. $n=0, \pm 1, \ldots, \pm N$. The number $N$ is determined by the frequency and the strength of the oscillation as $N>V_{1} / \hbar \omega[16]$.

By demanding non-trivial solutions of the coefficients $a_{n}, b_{n}, A_{n}$ and $t_{n}$ in Eq.(4), we obtain, after some tedious algebra, an equation which determines the Floquet energy $\varepsilon$ :

$$
F_{4} \frac{q_{0}}{k_{0}} \tan k_{0} a+F_{2}=\frac{F_{8} q_{0}+i F_{6} k_{0}^{\prime}}{F_{7} q_{0}-i F_{5} k_{0}^{\prime}}\left(F_{3} \frac{q_{0}}{k_{0}} \tan k_{0} a-F_{1}\right) e^{-2 q_{0}(b-a)} .
$$


Here $F_{i}\left(k_{0}, k_{0}^{\prime}, \omega, V_{1}\right)$ are functions of $k_{0}, q_{0}, k_{0}^{\prime}$ (see [12] for their complete forms), and hence are connected with the Floquet energy $\varepsilon$ (c.f. Eq. (3)). If the solutions $\varepsilon$ of Eq.(5) are complex (real) numbers, the corresponding Floquet states are metastable (stable) states. The non-decay probability $P(t)$, which is the probability of the particle still being trapped by the potential barrier at time $t>0$, is given by

$$
\begin{aligned}
P(t) & =\frac{\int_{0}^{b}|\Psi(x, t)|^{2} d x}{\int_{0}^{b}|\Psi(x, 0)|^{2} d x} \\
& =e^{2 \operatorname{Im}(\varepsilon) t / \hbar} \frac{\int_{0}^{b}\left|\Phi_{\varepsilon}(x, t)\right|^{2} d x}{\int_{0}^{b}\left|\Phi_{\varepsilon}(x, 0)\right|^{2} d x} \\
& \equiv e^{2 \operatorname{Im}(\varepsilon) t / \hbar} h(t),
\end{aligned}
$$

with $P(0)=1$. The imaginary part of the Floquet energy, which enters $P(t)$ via the factor $\exp (2 \operatorname{Im}(\varepsilon) t / \hbar)$, gives a measure of the stability of the system. Unlike the static case, however, here $P(t)$ is not a monotonic function of time, owing to the time-dependent function $h(t)$ after the exponential factor in Eq.(6). But since $h(t)$ is only a periodic function oscillating between two values which are of order one, the essential behavior of $P(t)$ at large times is still mainly governed by the exponential factor. Hence, as a useful measure of the non-decay rate of the particle in the well, we propose a coarse-grained non-decay probability $\bar{P}(t)$ defined as

$$
\bar{P}(t) \equiv e^{2 \operatorname{Im}(\varepsilon) t / \hbar}\langle h(t)\rangle
$$

where $\langle h(t)\rangle$ is the time-average of $h(t)$ over one period of oscillation (some graphs of $\bar{P}(t)$ are presented in [12]).

The coefficients $F_{i}\left(k_{0}, k_{0}^{\prime}, \omega, V_{1}\right)$ all approach to one in the limit $\alpha=V_{1} / \hbar \omega \rightarrow 0$. Hence in the limit $V_{1} \rightarrow 0$ or $\omega \rightarrow \infty$, Eq.(5) reduces to the corresponding equation for the case of static potential with potential $V_{0}$ in the region $a \leq x \leq b$, and the Floquet energy in this limit is just the (real or complex) eigenenergy of the static case. This is understandable, since in the limit $V_{1} \rightarrow 0$ the potential becomes static, and at high frequencies the particle in the well will only see a time-averaged barrier of effective height $V_{0}$.

\section{NUMERICAL RESULTS}

We now study numerical solutions of Eq.(5) with a specific potential. We take $a=1, b=2, V_{0}=15$ and $V_{0}^{\prime}=V_{0} / 2$ in the atomic units (a.u.) $\left(e=m_{e}=\hbar=1\right)$. In the static case this potential supports one bound state, with energy $E_{0} / V_{0}=0.232123$, and one metastable state, with complex energy $E_{1} / V_{0}=0.864945-0.00255261 i$. For the oscillating potential, we solve Eq.(5) in 2-sideband approximation, i.e. we take $N=2$. This is accurate enough for oscillating frequency $\omega \geq V_{1} / 2$.

Fig. 1 and 2 present the graphs of the real and imaginary parts of the Floquet energy $\left(\varepsilon / V_{0}\right)$ as a function of $\omega / V_{0} \geq 0.2$ with $V_{1}=0.1 V_{0}$ and $0.2 V_{0}$, respectively. We find that the solutions of Eq. (5) have the form $\varepsilon=\varepsilon_{0}+n \omega$ $(n=0, \pm 1, \pm 2, \ldots)$, with $\Re\left(\varepsilon_{0}\right)$ (the horizontal branch) lie close to the energies $E_{0}$ and $\Re\left(E_{1}\right)$ in the static potential. That is, these branches of $\Re(\varepsilon)$ emanate from either $E_{0}$ or $\Re\left(E_{1}\right)$ at $\omega=0$. Branches emerging from the same point have the same imaginary part. Numerical results show that, with the barrier oscillating, the stable state $\left(E_{0}\right)$ in the static case becomes unstable, and the unstable state $\left(E_{1}\right)$ will decay even faster. For simplicity, in Fig. (1a) and (2a) we show only six branches $(n=0, \pm 1, \pm 2$ and -3$)$ emerging from $\Re\left(E_{1}\right)$, and only the central branch $(n=0)$ and a subband $(n=-1)$ from $E_{0}$. As mentioned before, we only take solutions in the first Floquet zone, $\Re(\varepsilon)$ (modulo $\omega$ ), which are points under the line $\Re(\varepsilon)=\omega$.

From Fig. 1 and 2 we also see that a direct crossing occurs at frequency $\omega \approx \Re\left(E_{1}-E_{0}\right) / 2$ (point $c$ ). However, independent of the values of $V_{1}$, as $\omega$ approaches the frequency $\omega \approx \Re\left(E_{1}-E_{0}\right)=0.632822 V_{0}$, an avoided crossing $\left(e, e^{\prime}\right)$ between the real parts of the Floquet energies occurs. Fig. 2 indicates that larger values of $V_{1}$ only enhance the instability of the system and the repulsion between the two levels at avoided crossing. Thus as the frequency $\omega$ is increased, the state emanating from $E_{1}$ has Floquet energy with real part given by values along the path $a b b^{\prime} c d d^{\prime} e^{\prime} f$ (the dark dotted curve), while the real part of Floquet energy of the state emerging from $E_{0}$ lies along the path $\mathrm{cegg}^{\prime} h$ (the solid curve). The imaginary parts of these two paths are depicted in Fig. (1b) and (2b). One sees that an exchange of the imaginary parts takes place at the avoided crossing $e e^{\prime}$. Beyond the avoided crossing, the upper state becomes more stable than the lower state. This gives the possibility of stabilizing an unstable state by an oscillating field. We recall that as $\omega \rightarrow \infty$, Eq.(5) reduces to the one for the static potential. In the example considered here, the lower state supported by the static well is a stable bound state, and hence the unstable upper Floquet state can be made stable in the high frequency limit. Even more simply, the same aim can be achieved by adiabatically tuning down the amplitude $V_{1}$ just after the avoided crossing, as in this limit the potential becomes the static one. 
Finally, it is interesting to note here the rôle of amplitude $V_{1}$ of the oscillating barrier in the model (a detailed study is presented in [13]. As we have seen, the presence of a non-vanishing $V_{1}$ always makes the system less stable. However, if $V_{1}$ is reduced, an avoided crossing may turn into a direct one. In the present case, the avoided crossing $e e^{\prime}$ changes into a direct crossing for $V_{1} / V_{0}<0.03$. Conversely, increasing $V_{1}$ could change a direct crossing into an avoided crossing. At an avoided crossing, the imaginary parts of the Floquet energies cross, while the real parts do not. At a direct crossing, it is the real parts, not the imaginary parts, that cross. But the more (less) stable state has the tendency to become less (more) stable. This is evident from the Floquet energy at the direct crossing point $c$ in Fig. 1 and 2. These observations are consistent with the semiclassical results obtained in [11] by perturbative methods. Hence, by a combination of adiabatic changes of the frequency and the amplitude of the oscillating barrier, one can manipulate the stability of different states in a quantum potential: tune up $V_{1}$ until a direct crossing becomes an avoided one, increase $\omega$ so that the avoided crossing is passed, then reduce $V_{1}$ to make the potential static. In the process, two states in the well are interchanged.

\section{SUMMARY}

To summarize, our results show that an oscillating potential barrier generally makes a metastable system decay faster. However, the existence of avoided crossings of metastable states can switch a less stable state to a more stable one. If in the static well there exists a bound state, then it is possible to stabilize a metastable state by adiabatically changing the oscillating frequency and amplitude of the barrier so that the unstable state eventually cross-over to the stable bound state. Thus an time-dependent potential can be used to control the stability of a particle trapped in a well.

The model we present here has its barrier oscillating. One may also consider the same model with an oscillating bottom instead. However, this latter model turns out to have identical Floquet energy spectrum as the one presented here for the same frequency and amplitude of the oscillating field. This can be explained by a discrete transform which connects the equations of boundary conditions of the two models, and by a gauge transformation which maps the wave functions of the two models [13].

\section{Acknowledgments}

This work was supported in part by the National Science Council of the Republic of China through Grant No. NSC 94-2112-M-032-007. 
[1] G.Gamow, Z. Phys. 51, 204 (1928); R.W. Curney and E.U. Condon, Phys. Rev. 33, 127 (1929).

[2] For overviews on these developments, see eg.: L. Esaki, Proc. IEEE 62, 825 (1974); I. Giaever, Science 183, 1253 (1974); B.D. Josephson, ibid. 184, 527 (1974).

[3] S. Washburn, R.A. Webb, R.F. Voss, and S.M. Faris, Phys. Rev. Lett. 54, 2712 (1985); D.B. Schwarz, B. Sen, C.N. Archie, and J.E. Lukens, ibid. 55, 1547 (1985); M.H. Devoret, J.M. Martinis, and J. Clarke, ibid. 55, 1908 (1985).

[4] R. Tsu and L. Esaki, Appl. Phys. Lett. 22, 562 (1973); L.L. Chang, L. Esaki and R. Tsu, ibid. 24, 593 (1974).

[5] For a recent review, see eg.: N.B. Delone and V.P. Krainov, Multiphoton Processes in Atoms, Springer-Verlag, Berlin Heidelberg, 1994.

[6] A.H. Dayem and R.J. Martin, Phys. Rev. Lett. 8, 246 (1962); P.K. Tien and J.P. Gordon, Phys. Rev. 129, 647 (1963); B.J. Keay et al., Phys. Rev. Lett. 5, 4098 (1995).

[7] A.H. Guth, Phys. Rev. D23, 347 (1981); D. La and P.J. Steinhardt, Phys. Rev. Lett. 62, 376 (1989).

[8] E.W. Kolb and M.S. Turner, The Early Universe, Addison-Wesley, New York, 1990.

[9] M.P.A. Fisher, Phys. Rev. B37, 75 (1988).

[10] J.M. Martinis, M.H. Devoret, and J. Clarke, Phys. Rev. Lett. 55, 1543 (1985).

[11] D. Sokolovski, Phys. Lett. A132, 381 (1988).

[12] C.L. Ho and C.C. Lee, Phys. Rev. A71, 012102 (2005).

[13] C.C. Lee and C.L. Ho, Ann. Phys. 320, 175 (2005).

[14] J.H. Shirley, Phys. Rev. 138, B979 (1965).

[15] H. Sambe, Phys. Rev. A7, 2203 (1973).

[16] W. Li and L.E. Reichl, Phys. Rev.B60, 15732 (1999).

[17] F. Grossmann, T. Dittrich, P. Jung, and P. Hänggi, Phys. Rev. Lett. 67, 516 (1991).

\section{Figure Captions}

Figure 1. The Floquet energies $\left(\varepsilon / V_{0}\right)$ of the two metastable states versus the barrier oscillating frequency $\left(\omega / V_{0}\right)$ for $V_{0}=15$ a.u., $V_{0}^{\prime}=V_{0} / 2$ and $V_{1} / V_{0}=0.1$ in the atomic units (a.u.) $\left(e=m_{e}=\hbar=1\right)$. In (a) the real parts of the Floquet energies are shown in the first Floquet zone under the line $\Re(\varepsilon)=\omega$ (the straight line). The light dotted lines show how the different branches emanate from the two states in the static case (with $E_{0} / V_{0}=0.232123$, and $\left.\Re\left(E_{1}\right) / V_{0}=0.864945\right)$. In (b) the corresponding imaginary parts of the Floquet energies of the two states are plotted. The dotted curve corresponds to the state with real parts given along the path $a b b^{\prime} c d d^{\prime} e^{\prime} f$, and the solid curve corresponds to the state with real parts given along $c e g g^{\prime} h$.

Figure 2. Same plot as Fig. 1 for $V_{0}=15 a . u$., $V_{0}^{\prime}=V_{0} / 2$ and $V_{1} / V_{0}=0.2$. 


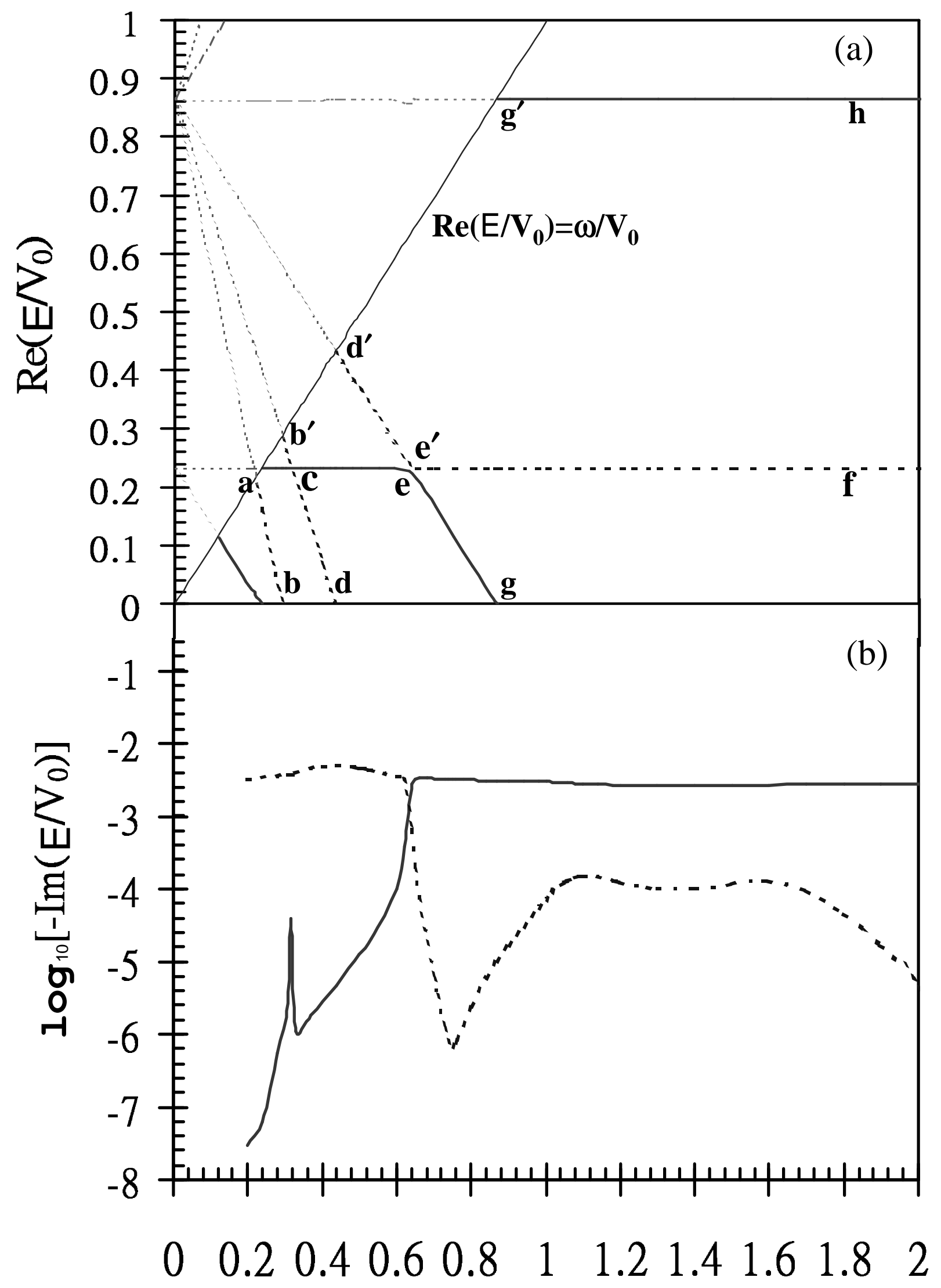

Figure 1

$\square / \mathrm{V}_{0}$ 


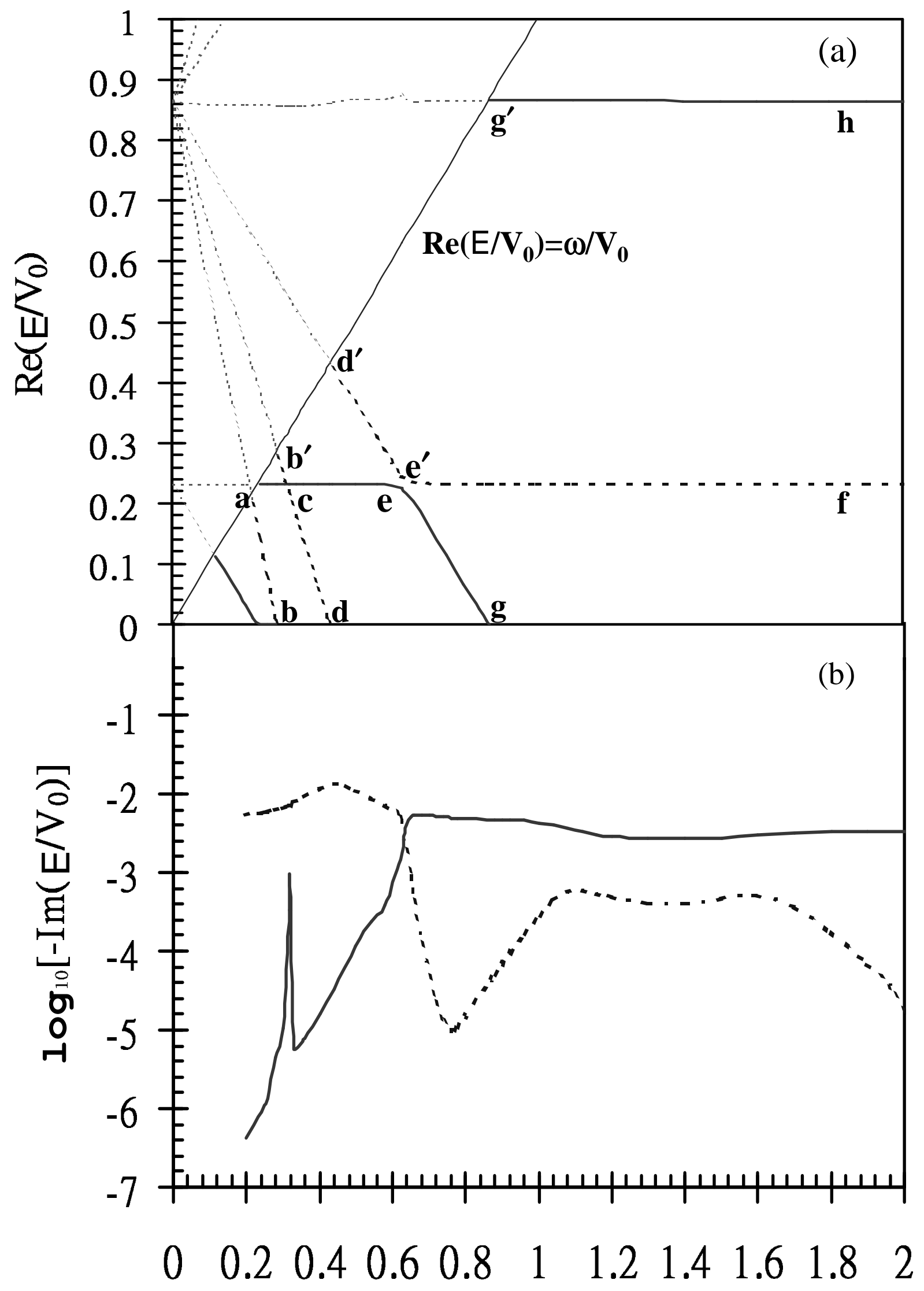

Figure 2 